\title{
IoT Smart Agriculture for Aquaponics and Maintaining Goat Stall System
}

\author{
Mohammad Kamil Rostam Effendi ${ }^{1}$, Murizah Kassim ${ }^{1 *}$, Norakmar Arbain \\ Sulaiman $^{1}$, Shahrani Shahbudin ${ }^{1}$
}

${ }^{1}$ Faculty of Electrical Engineering, Universiti Teknologi MARA, 40450 UiTM Shah Alam, Selangor, MALAYSIA

*Corresponding Author

DOI: https://doi.org/10.30880/ijie.2020.12.08.023

Received 12 April 2020; Accepted 19 June 2020; Available online 31 August 2020

\begin{abstract}
This research presents project development on smart farm and agriculture. The surge in global population is compelling a shift towards smart farm and agriculture practices. This coupled with the diminishing natural resources increase in unpredictable weather conditions makes food security a major concern for most countries. As a result, the use of the internet of things (IoT) and data analytics (DA) are employed to enhance the operational efficiency and productivity in the farm and agriculture sector. The objective is to design a prototype that used the internet of things on the farm and agriculture. Next is to have a monitoring and controlling or automation system that will benefit the farmer. Then collect all the data to be analyses on the rainfall, temperature, humidity, and light intensity. The methodology comprised of hardware, software, programming, sensors such as water sensor, light depending resistor sensor, temperature, and humidity sensor, and weight sensor for collected data. The result presents a prototype on aquaponics and goat stall that implement the concepts of the internet of things for monitoring, controlling, or automation system while data analytics is presented from all the sensors. Analytic data on the temperature, light intensity, humidity, and rainfall rate are analyzed. Surrounding temperatures are important for both plants and fish because if it too hot, they can die easily. The optimum light is needed by the plant for their photosynthesis process. Thus, by monitoring and collecting these parameters, data can be used for analyzing purposes. This project can benefit the agriculture and farm sectors in a larger or small scale.
\end{abstract}

Keywords: IoT, Smart Agriculture, Aquaponics; Goat Stall, Sensor, Node-RED, Arduino

\section{Introduction}

Farming plays an important role in today's world and it requires proper environmental and diet care. A smart system is needed to operate and monitor animals and plants remotely. The breeding and caring for farm animals could greatly be benefitted from a system capable of continuously monitoring the parameter that is affecting the system, combining the data, and reporting the obtained results to owners. A solution to this problem is to make a system that can be monitored on the internet through the dashboard. This solution can be achieved by using the Internet of Things (IoT). Some surveys have been explored how IoT platforms are developed and maintained from the perspective of the information system development process lifecycle. A detailed analysis of 63 approaches was presented which has accomplished by proposing an evaluation framework as a cornerstone to highlight the characteristics, strengths, and weaknesses of these approaches. The survey has provided insights of empirical findings, recommendations, and mechanisms for the development of qualityaware IoT platforms and also identified the important issues and gaps that need to be addressed [1]. Research has prescribed farming systems based on embedded systems, IoT, and wireless sensor networks for agriculture farm field and livestock farms. The description of systems with the electronic circuitry of the systems used network protocols and smart distant monitoring systems for PCs and Smartphones are discussed. This caters to the exponential growth of the human race of 

population, the conventional or ancient farming methods are becoming unable to cope with the growth with satisfaction. Thus, advanced farming methods are needed to approach the necessity in the future scopes of relevant technologies in smart farming [2]. IoT refers to a technology that tells that soon billions of devices will have stable connectivity and can be accessed from anywhere across the world. There are many components used to make IoT such as Wi-Fi module ESP8266 ESP-01, NODEMCU ESP8266 Wi-Fi [3], and Controller Board ESP-12 V2. These controllers are helpful in designed a small prototype system in many fields such as smart home [4], lighting control [5], healthcare [6], home security [7], and many more which help to simulate model before implemented at the real environment.

The project focuses on the development of smart farming which is expected to clear up the farming problems. Smart Farm could perceive any changed information derived from a semi-automatic microprocessor, alarming the notification to a connected personal computer. Farm monitoring could be conducted via application programs on smartphones for convenience use, time-saving, and reduce workload [8]. Thus, this project has merged the aquaponics system and goat stall system to make smart farming. Aquaponics is a bio-integrated food system that allows producing both plants and animals for consumption without requiring land to grow crops. Aquaponics can be defined as the integration of hydroponics, growing without soil, and aquaculture fish farming. Plants situated on water beds are grown with aquatic life, usually fish. The complex design allows for the waste products of one biological system to serve as nutrients for another [9]. The main components of developing aquaponic are plants, fish, and water pump. The fish and plants that need to select for the aquaponics system should have similar needs as far as temperature and ph. There is always be some compromised to the needs of the fish and plants but, the closer they match, the more successful of the project. Some of the suitable plants that can be cultivated are lettuce, basil, mint, and cabbage while the suitable fishes are tilapia, catfish, goldfish, and carp. The component used in this project for the aquaponics system is DHT22 Temperature and Humidity Sensor to measure temperature and humidity of the surrounding, Light Dependent Resistor to measure the intensity of light and Water Sensor to measure when there is rain. One of the most common uses for goat manure is as fertilizers. The goat stall system in this research is made to collect the goat manure to make fertilizer. Goat manure fertilizer can help gardeners produce healthier plants and crop yields. Goats not only produce neater pelletized droppings, but their manure does not typically attract insects or burn plants as does manure from cows or horses. Goat manure is virtually odorless and is beneficial for the soil. This manure contains adequate amounts of the nutrients that plants need for optimal growth, especially when the goats have bed installs. As urine collects in goat droppings, the manure retains more nitrogen, thus increasing its fertilizing potency. The sensors used in this system are HX711 Module with Weighing Sensor Load Cell to measure the weight of the manure and Ultrasonic Sensor to sense if there is an obstacle to push the manure into the container.

\begin{tabular}{|ll|}
\hline Nomenclature \\
\hline API & Application programming interface \\
AT & Attention \\
DA & Data Analytics \\
DHT & Digital-output humidity temperature \\
IoT & Internet of Things \\
L & Liter \\
LDR & Light-dependent resistor \\
LED & light-emitting diode \\
ma & Milligrams \\
MQTT & Message Queuing Telemetry Transport \\
TCP/IP & Transfer Control Protocol-Internet Protocol \\
\hline
\end{tabular}

\section{Literature Review}

\subsection{Aquaponics System}

An aquaponics system is a sustainable food production system where the growth of fish and plants happens when the natural bacterium that is needed by the plant in the nutrient-rich water comes from the waste excreted by the fish. As a response, the plants provide clean, nontoxic, and well-oxygenated water. Aquaponics comes from the combination of aquaculture and hydroponics. Aquaponics recycle the waste nitrogen from aquaculture discharge and increase Nitrogen Use Efficiency in crop productions [10]. An organic crop is produced by the recirculating system in aquaponics and preventing nitrogen losses via infiltration and surface leaching, which are the main problem for nitrogen losses in soilbased agriculture [11]. Aquaponics also engaged in measuring the polymerase chain reactions and next-generation sequencing to analyze the bacterial communities and their links to transform nitrogen [12]. There is also research where people are more willing to pay similarly for both aquaponics and soil-grown lettuce with some amount of money [13]. Research is also done for American Insular Pacific to evaluate a simple integrated lettuce and tilapia production system suitable to be developed. Aquaponics also provide sustainable food production with the lowest energy use and material waste comes from the thermal management of greenhouses [14]. Various nutrients that collected in the aquaponics systems have higher fish stocking density that caused a direct positive influence on the concentration (mg/L) [15]. Aquaponics is also used to analyze the production characteristics of alfalfa and mung bean sprouts grown in aquaponics and municipal tap water. In most of the physical parameters, the aquaponics and tap treated sprouts were found to be different [16]. Also, 
the efficiency and output of a commercial aquaponics system in research that has been done on an aquaponics life cycle assessment. The research evaluated an innovative method for growing local fish and lettuce [17].

\subsection{Goat Stall System}

The goat stall system is used to collect the goat's manure. Using goat manure in garden beds can create optimal growing conditions for plants. However, a goat with good health is important to make sure that a good quality of fertilizer is produced. The goat health management system has implemented a routine monitoring standardization, applicable and integrity electron case file, disease prevention systematization [18]. There is some study that has demonstrated the performance of Sweet Corn which has applied with Goat Manure and Bio-N. The field experiment was carried out in a randomized complete block design where different treatment rates of GM improved significantly all the growth parameters of sweet corn while BN only increased significantly. The performance of sweet corn was remarkably affected by GM. No significant GM and BN interaction was observed. This study provides relevant information that could help sweet corn farmers to avail of an alternative scheme in generating a good harvest without sacrificing soil sustainability [19].

\section{Methodology}

\subsection{Flowchart}

The methodology presents a flowchart on the prototype of the aquaponics and goat stall system as in Figure 1 and Figure 2. Figure 1 shows the flowchart process of the aquaponics system. The concept of smart in these systems presents an implemented of IoT system that received information from all the sensors. First, the system is connected to the internet, and then all the sensors have gathered information on the light intensity, temperature, humidity, and rainfall. Then it is published through the MQTT broker and receive by Node-RED to be displayed on the dashboard. Here, the user can monitor all the information that was collected from the sensor. This system designed to decide if the light intensity is too high or it is raining, then the shade is turned on to keep optimal light intensity receive by the plant and protect the plant from receiving too much water from the rain. Figure 2 shows the processes that occur in the goat stall system. In this system, the goat's manure or waste has been collected. First, when the process of collecting is starting, the door is automatically open for the goat to come out. Then, a sensor is checked if there any more goat or person inside it. When there is no one inside the stall. The door is closed to prevent anything to enter it during the collecting process. Next, a motor is turned on to push all the manure into a big container. Inside the container, a sensor is installed to check the weight of the manure. The display will alert of the manure when it is full. The development prototype of these systems is hopefully to help other farmers to make their work easier.

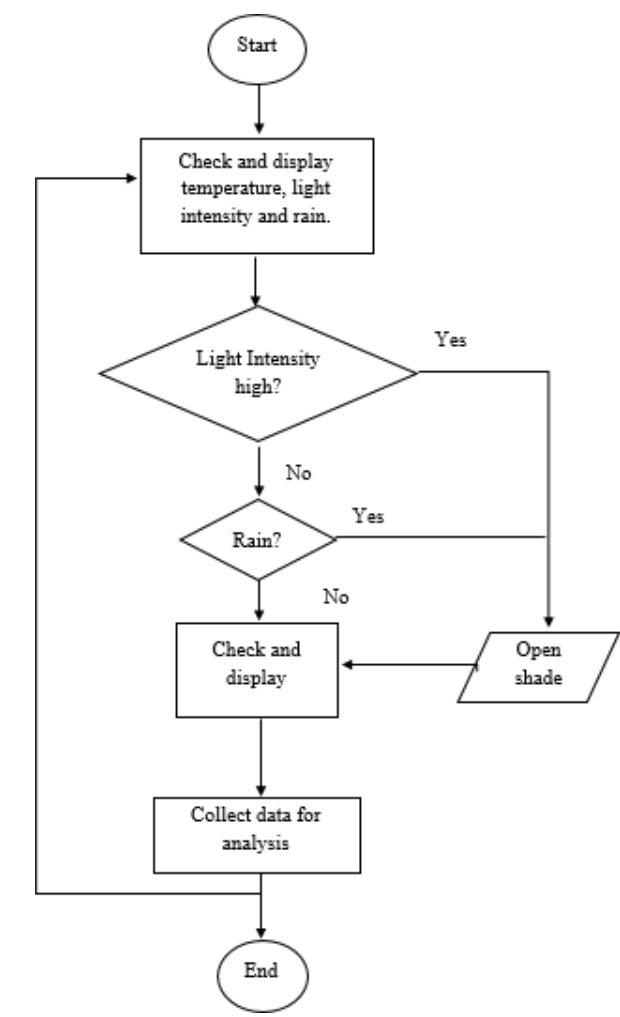

Fig. 1 - Flowchart of aquaponics system

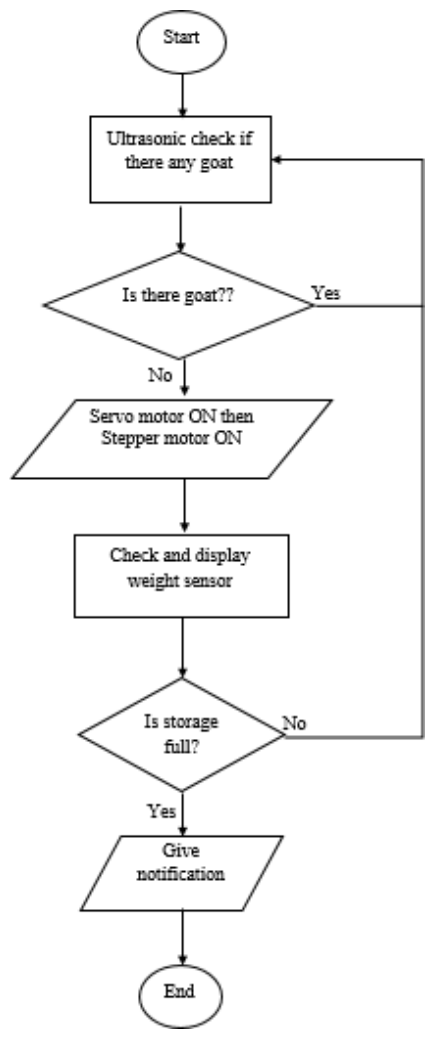

Fig. 2 - Flowchart of goat stall 


\subsection{System Design}

Figure 3 shows the prototype of this project system design in a block diagram. Sensors that are used in projects like water sensor, temperature and humidity sensor, light sensor, weight sensor, and ultrasonic sensor are connected to the ESP8266-01 as input. ESP8266-01 acts as the communication medium that sent data to the microcontroller used which is Arduino and displays it on a dashboard using Node-RED. The microcontroller received the signal from the ESP8266-01, and then it controlled the hardware outputs which are stepper motor and servo motor. First, the Arduino needs to be coded with identified instruction to all hardware as the projectrequired.

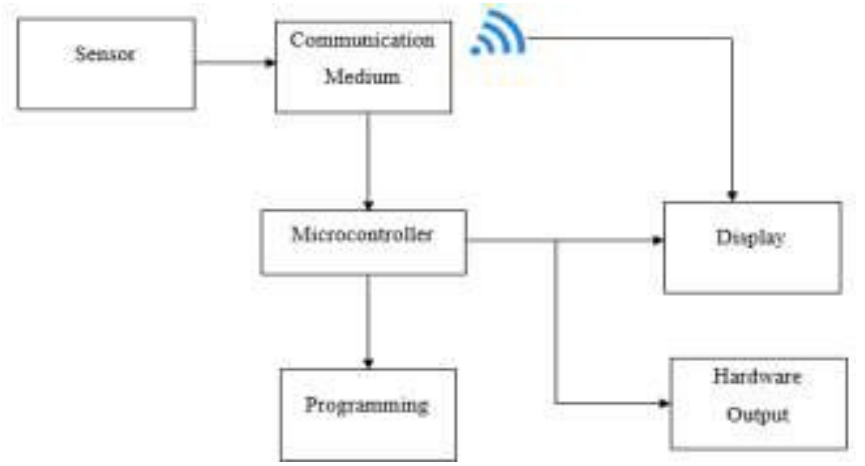

Fig. 3 - Block diagram

\subsection{Design Structure}

Figure 4 shows a designed structure for the aquaponics system. The input port has been designed with three sensors which are DHT22 sensor, water sensor, and LDR sensor while only stepper motor was used as the output part. Arduino Mega acts as controller and ESP8266-01 which added with a Wi-Fi module that connects the system. The wifi features for internet communication which displayed all parameters on the Node-RED dashboard online. Data collections also are saved to the Excel datasheet. Figure 5 shows the Goat stall design structure that presents Arduino UNO that acts as a controller that received data from the input which is the load cell weight sensor and ultrasonic sensor. Then the controller sent a command to the output device like stepper motor and servo motor.

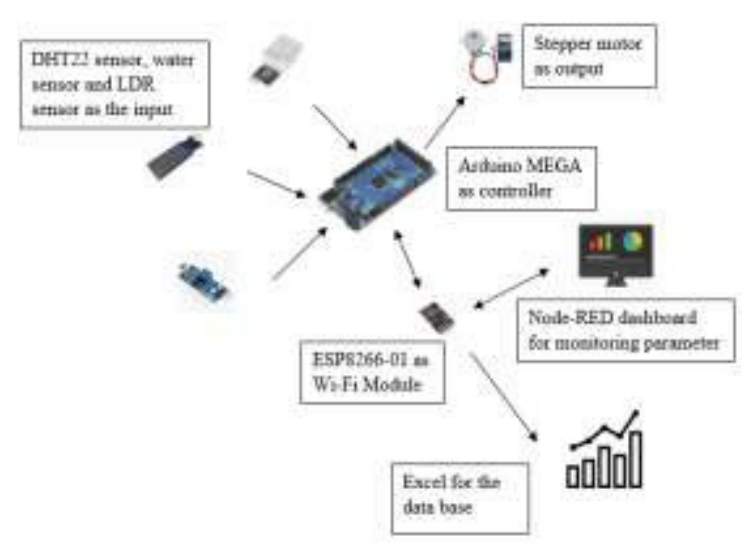

Fig. 4 - Aquaponics design structure

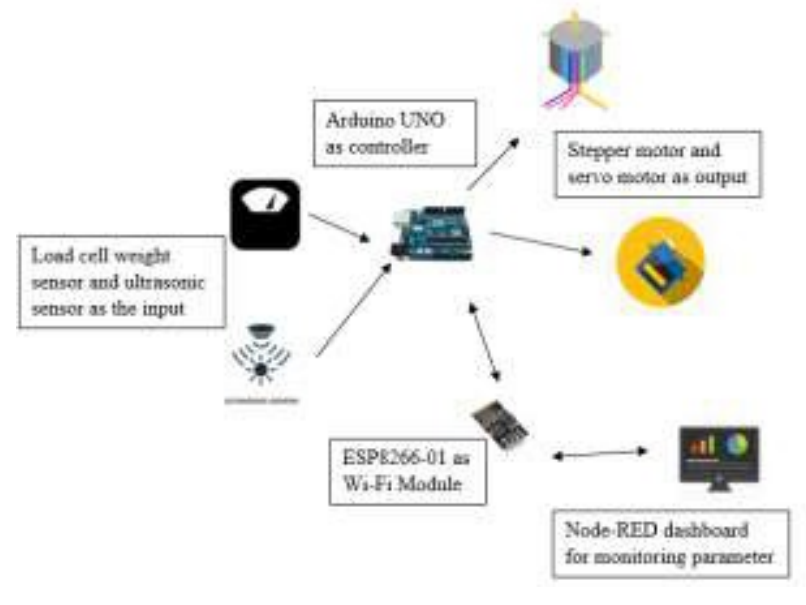

Fig. 5 - Goat stall design structure

\subsection{Hardware and Software}

Controller and Software: Arduino UNO and Arduino Mega are used in this project. Both microcontrollers are the brain that controls all the sensors and motor. Arduino is more efficient as it does not have separate pieces of hardware to load new code into the microcontroller, it simply used a USB cable to upload it and the software for Arduino uses a simplified version of $\mathrm{C}++$ language to program it. While Arduino IDE (Integrated Development Environment) is the software for Arduino [20]. The software is used to program a set of instruction that has informed the hardware of what and how to do it. A sensor is an important part of making the system smart. Many sensors are used today to replaced humans in which sensors can collect data from various things such as temperature, humidity, motion, $\mathrm{pH}$ value, distance, and many more to be used in monitoring and controlling the smart systems [21]. Sensors that were used in this project are a water sensor that has detected the raining or vice versa. Temperature and humidity sensor called DHT22 for measuring surrounding 
temperature and humidity. Next is the light depending resistor (LDR) as a sensor that measures light intensity. The ultrasonic sensor also was used in the goat stall system. It is usually used to measure distance. However, in this project, we used it to check if there anything inside the stall during the collecting process. Lastly, a weight sensor used to indicate the weight of the goat's manure that has been collected.

Communication medium: The Internet was used as the communication medium in the project. The device for using the internet as the medium is called the ESP8266-01 module. The ESP8266 is a low-cost Wi-Fi microchip with full TCP/IP stack and microcontroller capability produced by Shanghai-based Chinese manufacturer, Expressive Systems. ESP8266 module has allowed users to send and receive data and it works on AT commands that run automatically [22]. Database and Analysis: Node-RED was used in this project to display data and making analysis. Node-RED is a programming tool for wiring together hardware devices, APIs, and online services in new and interesting ways [23]. It provides a browser- based editor that makes it easy to wire together flows using the wide range of nodes in the palette that can be deployed to its runtime in a single click. Node-RED provides a browser-based flow editor that makes it easy to wire together flows using the wide range of nodes in the palette. Flows can be then deployed to the runtime in a single click. JavaScript functions can be created within the editor using a rich text editor. A built-in library allows you to save useful functions, templates, or flows for re-use.

\section{Result and Analysis}

A Prototype on both aquaponics and goat systems has been developed and all the data are collected from all the sensors. For this project, the aquaponics prototype has been placed indoor because of a lack of suitable space outside my house and a high probability of stray cats damaging the prototype. A dashboard was designed using Node-RED software. Figure 6 shows the flow of nodes that are connected to contact flow in displaying data. Figure 7 shows a Node-RED dashboard that displayed data collection from sensors and plotted in graphical graphs. The MQTT broker is published and subscribed to send and receive data from the sensors then only data is displayed on the Node-Red dashboard. Backup data is saved in excel datasheet .csv file which also can be view online. All data from the DHT22 sensor, water sensor, and LDR sensor are monitored on the dashboard in identified parameters against time.

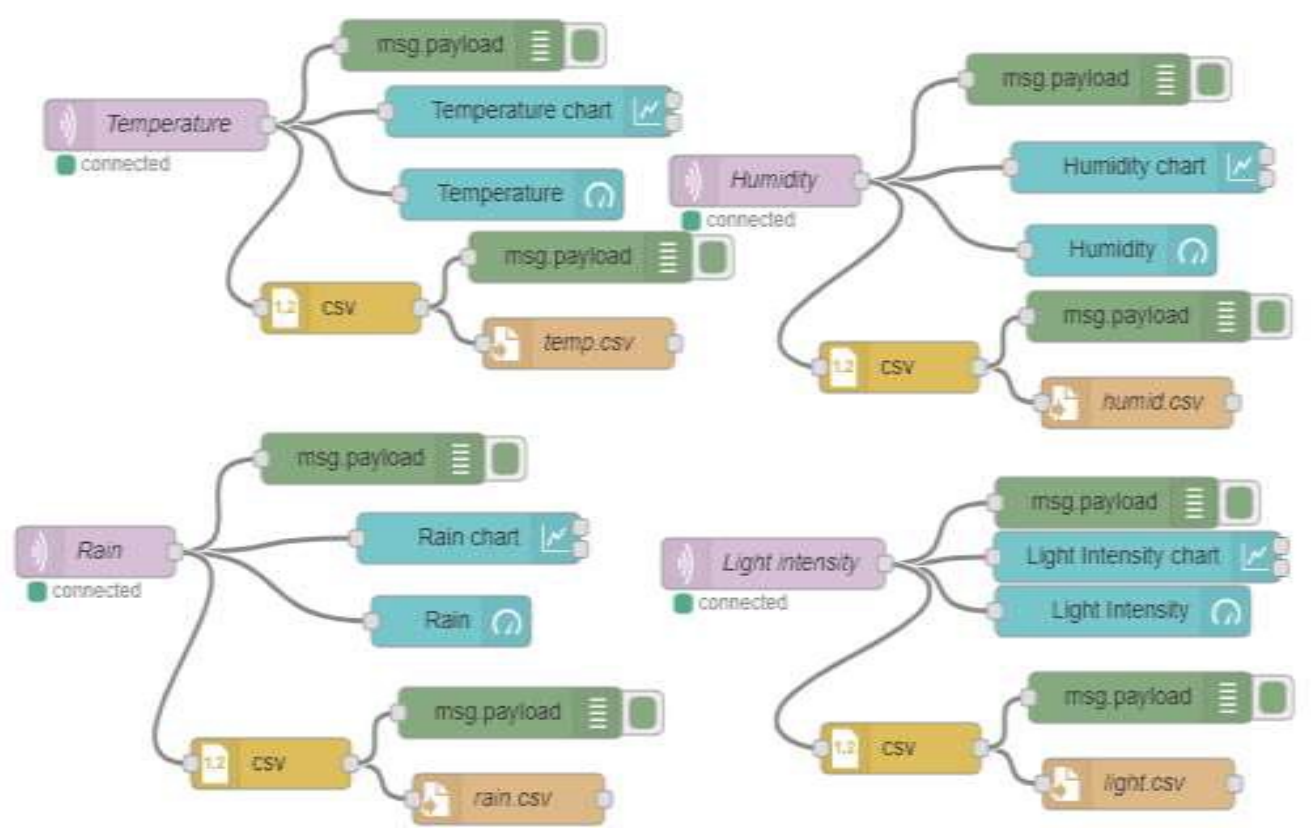

Fig. 6 - Node-RED flow 


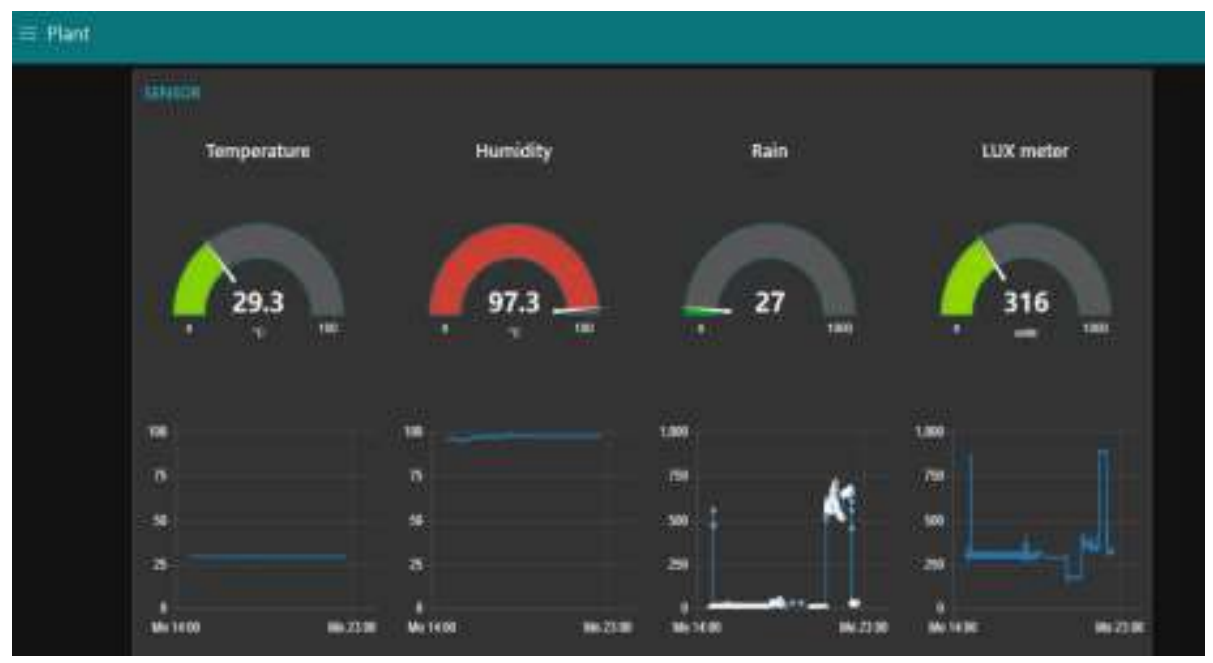

Fig. 7 - Node-RED dashboard

\subsection{Project Prototype}

Figure 8 shows all important labels in the alphabet from A to $H$ that shows the labels in Table 1 . The prototype of the aquaponics system designed with three (3) sensors which are DHT22 for measuring temperature and humidity (E), water sensor (G), and LDR (F). The prototype was placed indoor due to several technical problems. Label A is shaded that has filtered light from the sun while label B is the water pump that connects water form fish container into the plant container. Label $\mathrm{C}$ is the container for the fish and label $\mathrm{H}$ is the container for the plant. Lastly, label $\mathrm{D}$ is a box that contains Arduino Mega and ESP8266-01 which is the brain of this system. In designing and assembling this prototype, several problems have been encountered that need to be solved such as the use of plastic bottles to hold the plant. However, the plant still needs some soil in the bottle to hold it more firmly. The hole for the tube needs to be sealed properly to avoid possible leakages.

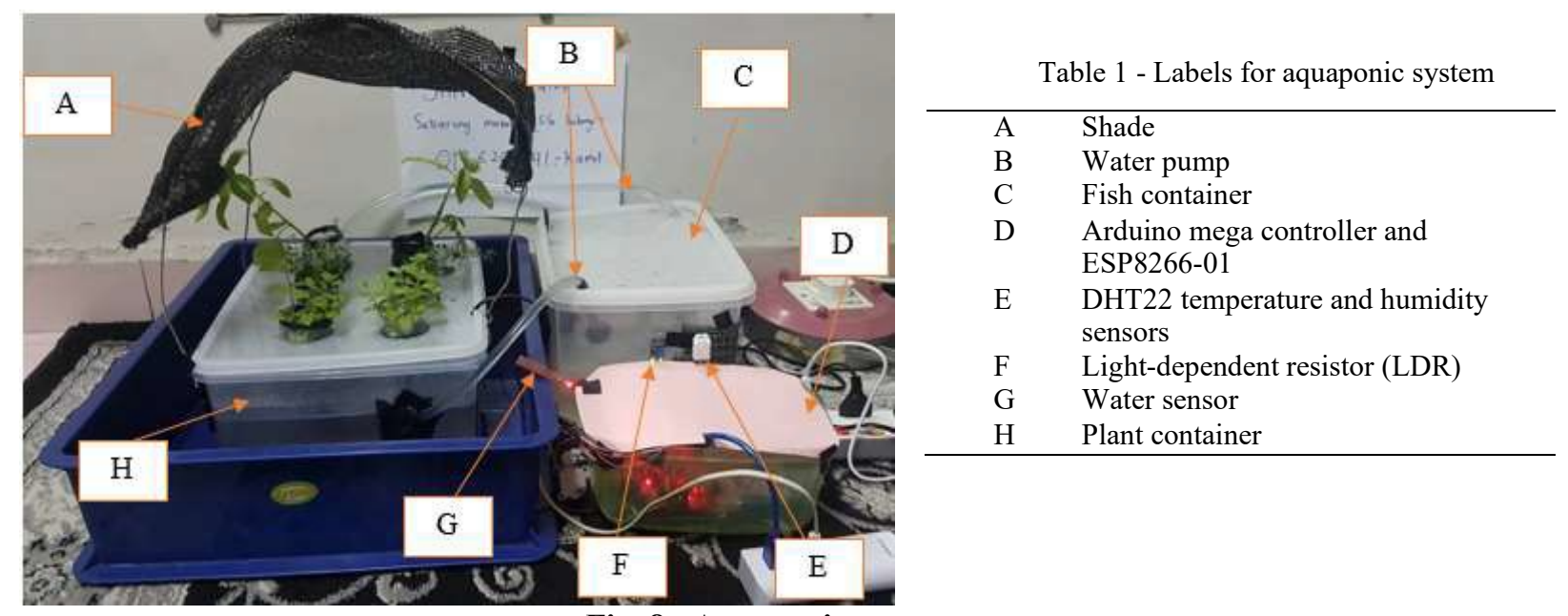

\section{Fig. 8 - Aquaponic system prototype}

Figure 9 shows the circuit designed for the goat stall system. ESP8266-01 is a label with A is used to allow the Arduino to send data into Node-RED via the internet. Table 2 shows all labels which Label B is the Arduino Uno that acted as a microcontroller to control the entire component that was connected to it. The ultrasonic sensor is labeled as $\mathrm{C}$ is to check condition in the stall and the process of the collection started by turning on the stepper motor as label D. The servo motor is label E acted as a mechanical part that will open and close the door. Lastly, the load cell of the weight sensor as labeled $\mathrm{F}$ is used to measure the weight of goat manure collected in time. 


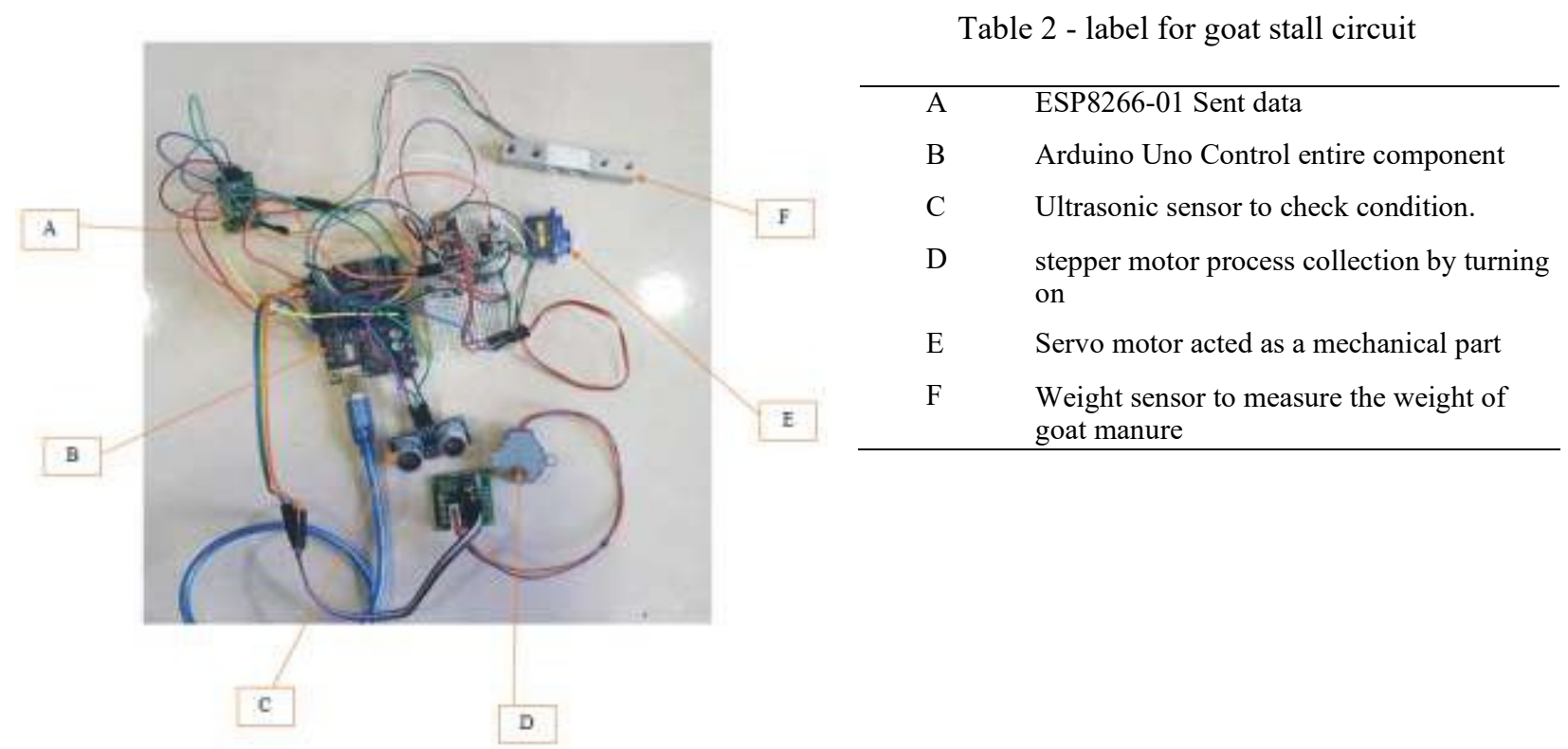

Fig. 9 - Goat stall system circuit

\subsection{Data Collection and Analytics}

Data collection from all sensors has been collected and analyzed in time with parameter sets in all sensors. The data is collected inside the house from 2.30 p.m. until 10.30 p.m. a day. Several factors have affected the data accuracy which is the connection to the internet itself. Wi-Fi connection in the house was used in this project and it is identified that the connection was not quite stable during data collection. On the other hand, the MQTT broker server faces some intermittent connections by using an open-source platform which is free that many people possible to be online. Water and LED also was used to shows the sensor is worked because this project was placed indoor where raining simulation is done. LED is used to monitor the increased or decreased light intensity.

\section{- Temperature Analysis}

Figure 10 shows that the temperature analysis taken in time. The standard temperature for an aquaponics system is between $21^{\circ} \mathrm{C}$ to $29^{\circ} \mathrm{C}[24,25]$. This value is analyzed as a suitable temperature for both plants and fish. Some value around $27.5^{\circ} \mathrm{C}$ also is identified if the prototype is placed inside the house[26]. Thus, the temperature does not vary so much compare to the temperature if it is placed outdoor. This is important where more aquaponic plants need to cover with shade, Figure 11 shows the difference in temperature analysis taken during day and night.

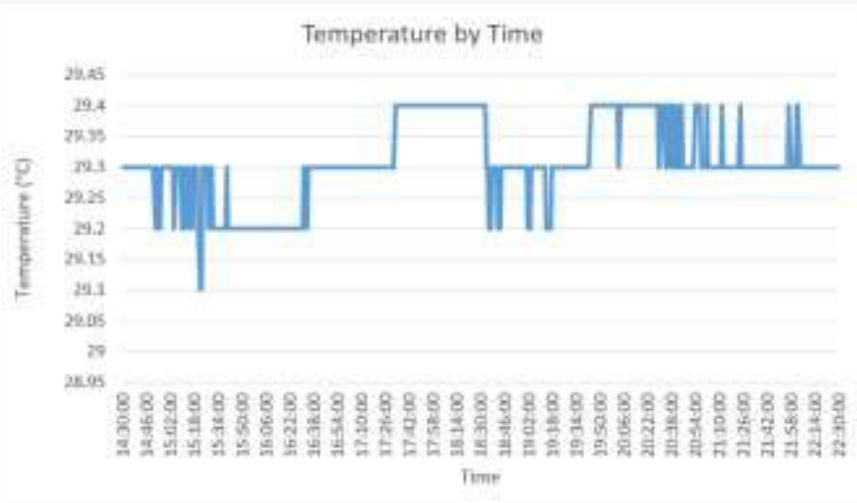

Fig. 10 - Temperature chart from the DHT22 sensor

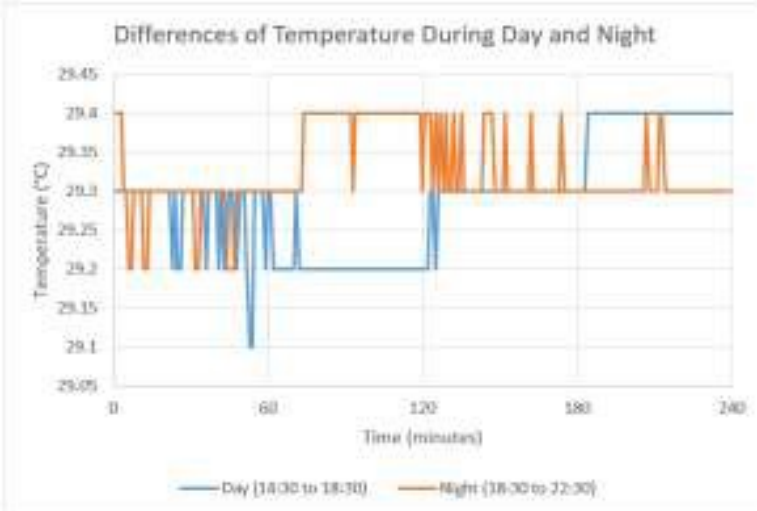

Fig. 11 - The difference of temperature during day and night 


\section{- Humidity Analysis}

Figure 12 shows the highest value of humidity is $96.3 \%$ and the lowest is $95.7 \%$. It is identified that Malaysia weather has high humidity which night humidity shows higher that at the day. Figure 13 shows the comparison of humidity that was taken for 4 hours during the day from 14:40 until 18.30 and a 4 hour taken during the night from 18:30 until 22:30. The analysis shows there are some small differences in humidity readings for both times which shows humidity does not vary so much. This time is taken for plants that need more sunlight in the afternoon in comparison with humidity level and nighttime is taken where there is no sunlight to theplants.

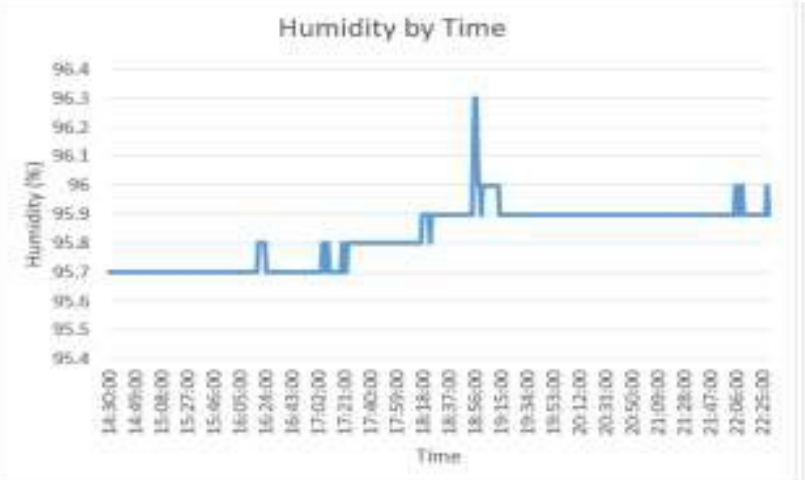

Fig. 12 - Humidity analysis from the DHT22 sensor

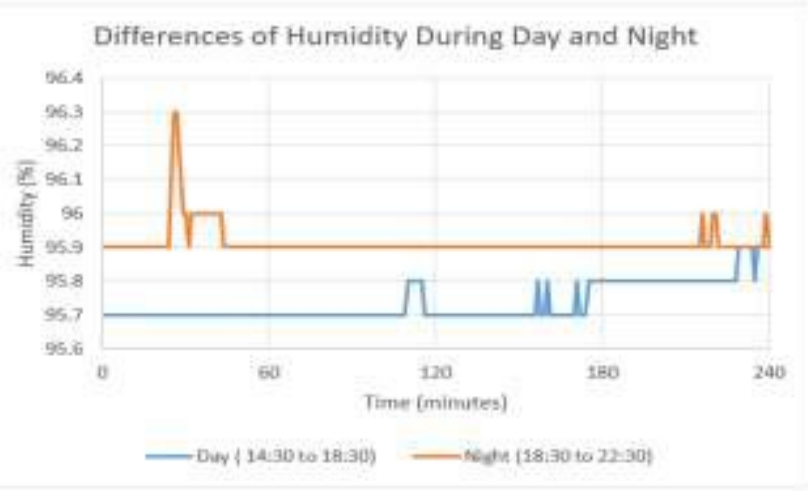

Fig. 13 - Humidity analysis during day and night

\section{- Rain Analysis}

Figure 14 shows the rainfall occurs during the testing of the prototype. Figure 10 shows the simulations done started at 7.40 p.m. until 9.40 p.m. which the value increases significantly because the water sensor detects water. Figure 15 shows the comparison of rainfall for 4 hours during the day from 14:40 to 18.30 time and 4 hours during the night from 18:30 until 22:30 time. During the day, the weather is good without any rain as it shows a constant 0 values. However, during the night the value increase above 600 indicates that it is raining. Thus, the stepper motor action is to close the shade when detected of raining. This step of shade also helps to protect plants for too much watering for some identified plants.

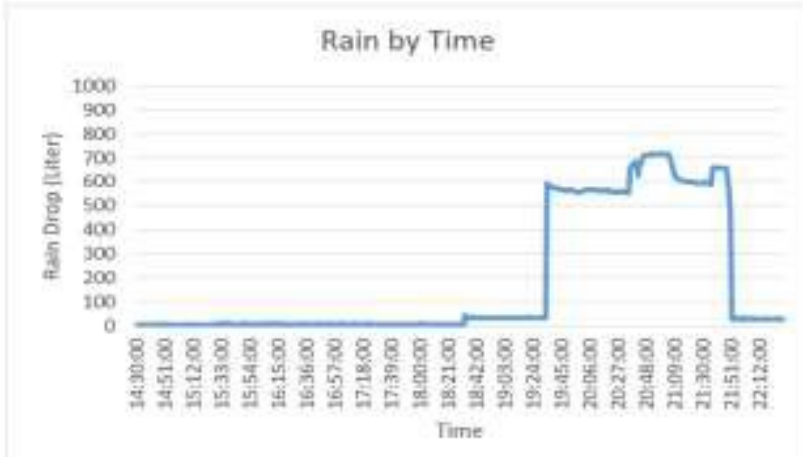

Fig. 14 - Rainfall analysis data

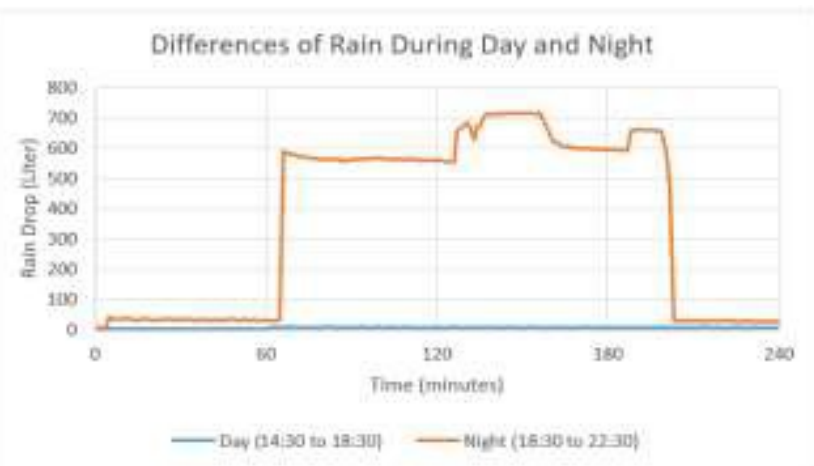

Fig. 15 - The difference of rainfall during day and night

\section{- Light Intensity (Lux) Analysis}

Figure 16 shows when the LDR sensor detects light. The graph shows a variation of value from 2.30 p.m. until 10.30 p.m. indicates that the light intensity increases and decreases during that time. The tested is done in with normal house light which the value is constant around 300 light intensity. Then, it is tested with LED near the system; the value drops below 200 units indicate there is high light intensity. However, when all the light is turned off, the value increased very high up to 900 units. Most of the plants need 12 to 16 hours of light every day for the photosynthesis process. The low light intensity has slowed down the photosynthesis process. Thus, it needs more hours of light in a day. 


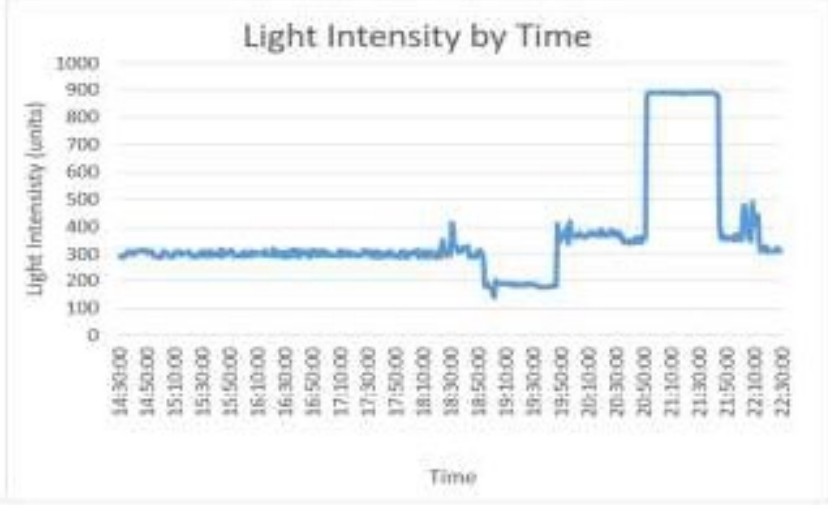

Fig. 16 - Light intensity analysis

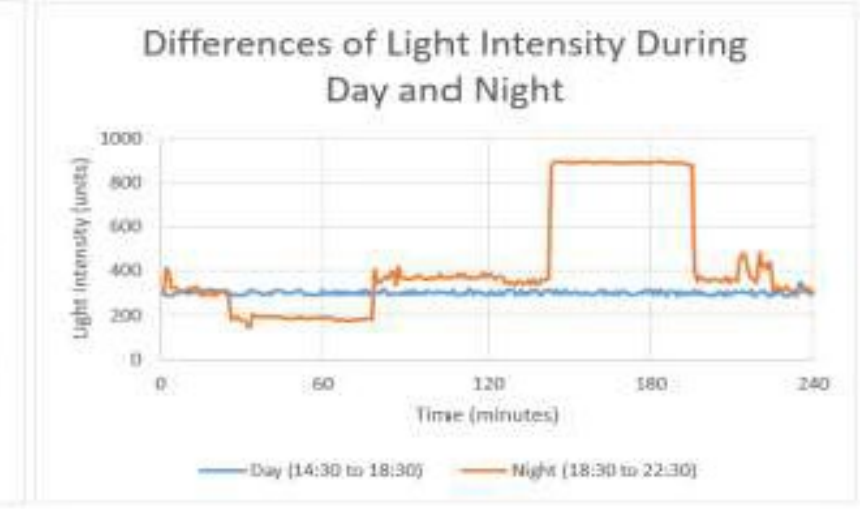

Fig. 17 - Differences in light intensity

Figure 17 shows the comparison of light intensity which 4-hours during the day from 14:40 until 18.30- and 4-hours during the night from 18:30 until 22:30. During the day, the light intensity is constant at around 300 units because there is no one in the house. However, during the night the value decrease below 200 indicates high light intensity due to the LED that has been used near the plant. Then the value increases above 800 because all the light has been turned on.

\section{- Collected Manure Data}

Figure 18 presents the value of the goat manure that has been collected. Every day the same process is kept on repeated until it reached maximum weight. After the maximum weight is collected then the goat manure is transferred for the next process for the user back as organic fertilizer for the plants. This process presents the process of growth of the plant by using goat manure production.

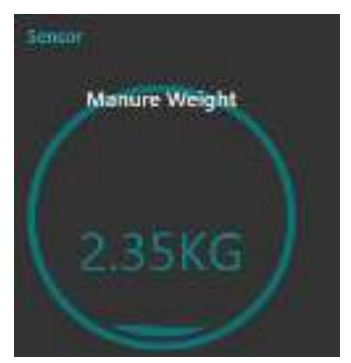

Fig. 18 - Manure weight from the weight sensor

\section{Conclusion}

It is concluded that this research has succeeded in designing a prototype for aquaponics systems and goat stall systems. In the aquaponics prototype, it has both a fish container and plant container that are connected. By using this prototype, water usage can be optimized because fish wastage has provided the nutrient for the plant while the plant has been filtered the water for the fist. Both systems have sensors that measure an important parameter. With these parameters, it can be displayed on the user dashboard for monitoring purposes. Thus, the user does not need to go to the farm to check on the plant, fish, and goat manually. Data analytic in real-time is presented for all the important parameters as it been collected from the sensor and sent through the internet using the ESP8266-01 module. The data collection has been analyzed and based on the development still some drawback exists, and it is to be improved for its system and the product quality. The design of this system has been registered as Smart Kebun 1.0 and future work that can be implemented to improve this system will be called Smart Kebun 2.0. The enhancement of Smart Kebun 2.0 will include integrating the product storage, processing, and packaging, and transporting the product. The implementation of Smart Kebun 2.0 will monitor the quantity and quality of the product in the storage. The analysis of the processing and packaging area for a better-quality product also has becoming and important actors, concept, and modeling process for smart agriculture in developing and coherent with IR4.0 technology development. This research is significant in the production line which will have a more systematic schedule in transporting the product for smart agriculture.

\section{Acknowledgment}

This author would like to thank the Enabling Internet of Things Technologies, Research group at the Faculty of Electrical Engineering, Universiti Teknologi MARA, UiTM Shah Alam for the support materials and lab for this research. 


\section{References}

[1] Fahmideh, M., and Zowghi, D. (2020). An exploration of IoT platform development. Information Systems 87, 101409.

[2] Mahbub, M. (2020). A Smart Farming Concept Based on Smart Embedded Electronics, Internet of Things and Wireless Sensor Network. Internet of Things, 100161.

[3] Athawale, S., Solanki, M., Sapkal, A., Gawande, A., and Chaudhari, S. (2020). An IoT-Based Smart Plant Monitoring System. In Smart Computing Paradigms: New Progresses and Challenges. (Springer), pp 303-310.

[4] Zainal Arifin, M.A., Kassim, M., Mahmud, A.R., and Izwan Suliman, S. (2019). Automation security system with laser lights alarm on web pages and mobile apps. In ISCAIE 2019 - 2019 IEEE Symposium on Computer Applications and Industrial Electronics. pp 287-292.

[5] Muhamad, W.N.W., Mohd Anuar, A.S., Sarnin, S.S., Mohamad Azreen, M., Idris, A., and Kassim, M. (2019). Intelligence Lighting Control System with Precise Dimming and Correlated Color Temperature. In IEEE Region 10 Annual International Conference, Proceedings/TENCON. pp 1235-1240.

[6] Rahman, R.A., Aziz, N.S.A., Kassim, M., and Yusof, M.I. (2017). IoT-based personal health care monitoring device for diabetic patients. In ISCAIE 2017 - 2017 IEEE Symposium on Computer Applications and Industrial Electronics. pp 168-173.

[7] Jaafar, A., Kassim, M., Haroswati, C.K., and Yahya, C.K. (2017). Dynamic home automation security (DyHAS) alert system with laser interfaces on webpages and windows mobile using raspberry PI. In 2016 7th IEEE Control and System Graduate Research Colloquium, ICSGRC 2016 - Proceeding. pp 153-158.

[8] Pu'ad, M.F.M., Sidek, K.A., and Mel, M. Water Quality Monitoring System for Aquaponics and Fishpond Using Wireless Sensor Network.

[9] Wahl, S. (2010). Discovering Aquaponics. Aquaponics Journal.

[10] Wongkiew, S., Popp, B.N., Kim, H.-J., and Khanal, S.K. (2017). Fate of nitrogen in floating-raft aquaponic systems using natural abundance nitrogen isotopic compositions. International Biodeterioration \& Biodegradation 125, 2432.

[11] Shelton, R.E., Jacobsen, K.L., and McCulley, R.L. (2018). Cover crops and fertilization alter nitrogen loss in organic and conventional conservation agriculture systems. Frontiers in plant science 8, 2260.

[12] Paudel, S.R. (2020). Nitrogen transformation in engineered aquaponics with water celery (Oenanthe javanica) and koi carp (Cyprinus carpio): Effects of plant to fish biomass ratio. Aquaculture 520, 734971.

[13] Short, G., Yue, C., Abbey, M., Anderson, N., Phelps, N., Venturelli, P., and Vickers, Z. (2018). Consumer preferences for aquaponic produce: Implications from an experimental auction. Agribusiness 34, 742-755.

[14] Amin, M.T., and Kissock, J.K. (2016). Dynamic modeling and verification of an energy-efficient greenhouse with aquaponics. In ASME 2016 10th International Conference on Energy Sustainability collocated with the ASME 2016 Power Conference and the ASME 2016 14th International Conference on Fuel Cell Science, Engineering and Technology. (American Society of Mechanical Engineers Digital Collection.

[15] Manduca, L.G., da Silva, M.A., de Alvarenga, É.R., de Oliveira Alves, G.F., de Araújo Fernandes, A.F., Assumpção, A.F., Cardoso, C.C., de Sales, S.C.M., de Alencar Teixeira, E., and e Silva, M.d.A. (2020). Effects of a zero exchange biofloc system on the growth performance and health of Nile tilapia at different stocking densities. Aquaculture 521, 735064.

[16] Davis, K.F.C. (2016). Effect of Irrigation Water on Growth and Physical Characteristics of Alfalfa (Medicago Sativa) and Mung Bean (Vigna Radiata) Sprouts.

[17] Hollmann, R.E. (2017). An Aquaponics Life Cycle Assessment: Evaluating an Inovative Method for Growing Local Fish and Lettuce.(University of Colorado at Denver).

[18]Zobel, G., Neave, H.W., and Webster, J. (2019). Understanding natural behavior to improve dairy goat (Capra hircus) management systems. Translational Animal Science 3, 212-224.

[19] Talip, O.S., and Sison, L.C. (2017). Performance of Sweet Corn, Zea mays L. saccharata Applied with Goat Manure and Bio-N®. Journal of Multidisciplinary Studies 6, 114-137.

[20] Anuar, F.F., Zain, B.A.M., and Al-Shaibani, N. (2018). Comparative study on flexible link aerator using arduino programming and dissolved oxygen meter. International Journal of Integrated Engineering 10, 1-5.

[21] Kassim, M., Haroswati, C.K., Yahaya, C.K., and Ismail, M.N. (2010). A prototype of Web Based Temperature Monitoring system. In ICETC 2010 - 2010 2nd International Conference on Education Technology and Computer. pp V5266-V5270.

[22] Singh, P., and Saikia, S. (2016). Arduino-based smart irrigation using water flow sensor, soil moisture sensor, temperature sensor and ESP8266 WiFi module. In 2016 IEEE Region 10 Humanitarian Technology Conference (R10-HTC). (IEEE), pp 1-4.

[23] Miskon, M.T., Hasry, M.A.H., Rosle, M.S., Danial, A., and Hidayah, A. (2019). IoT-Enabled Electric Socket Test Bed Utilizing IFTTT and Node-Red. Journal of Advanced Research in Engineering Knowledge 9, 6-13. 
[24]Kumar, N.H., Baskaran, S., Hariraj, S., and Krishnan, V. (2016). An Autonomous Aquaponics System Using 6LoWPAN Based WSN. In 2016 IEEE 4th International Conference on Future Internet of Things and Cloud Workshops (FiCloudW). (IEEE), pp 125-132.

[25] Delaide, B., Delhaye, G., Dermience, M., Gott, J., Soyeurt, H., and Jijakli, M.H. (2017). Plant and fish production performance, nutrient mass balances, energy and water use of the PAFF Box, a small-scale aquaponic system. Aquacultural Engineering 78, 130-139.

[26] Mamatha, M., and Namratha, S. (2017). Design \& implementation of indoor farming using automated aquaponics system. In 2017 IEEE International Conference on Smart Technologies and Management for Computing, Communication, Controls, Energy and Materials (ICSTM). (IEEE), pp 396-401. 